First publ. in: Journal of Atmospheric and Oceanic Technology 22 (2005), 11, pp. 1821-1828

\title{
Application of Coherent ADCP for Turbulence Measurements in the Bottom Boundary Layer
}

\author{
ANDREAS LORKE \\ Applied Aquatic Ecology, EAWAG, Kastanienbaum, Switzerland, and Environmental Physics, Limnological Institute, University of \\ Constance, Constance, Germany \\ AlfRed WÜEst \\ Applied Aquatic Ecology, EAWAG, Kastanienbaum, Switzerland
}

(Manuscript received 2 June 2004, in final form 20 December 2004)

\begin{abstract}
This paper presents a novel approach for estimating the rate of turbulent kinetic energy dissipation from pulse-coherent acoustic Doppler current profiler (ADCP) measurements using the inertial dissipation method. Although the inertial dissipation technique is widely accepted and used in oceanographic and atmospheric research, its application to ADCP data is limited by the loss of directional information for high-wavenumber velocity fluctuations. However, measurements in the bottom boundary layer of a lake revealed astonishing agreement between dissipation rates estimated from temperature microstructure profiles and those estimated by applying the inertial dissipation method to data from two different brands of ADCPs.
\end{abstract}

\section{Introduction}

In this paper we test the applicability of the inertial dissipation method (IDM) for estimating profiles of dissipation rates $\varepsilon$ of turbulent kinetic energy from the three-dimensional current velocities measured by conventional acoustic Doppler current profilers (ADCPs) operated in pulse-coherent mode. The IDM is based on the existence of an inertial subrange in the wavenumber spectrum of velocity fluctuations and is a wellestablished and widely accepted method to estimate dissipation rates from measured time series of two- or three-dimensional current velocities. For technological reasons, however, applications have been restricted so far to single-point measurements or to data from instrument arrays.

ADCPs are capable of measuring profiles of the current velocity by applying run-time windowing of backscattered acoustic pings along three or four beams pointing in different directions. Whereas normal-mode

Corresponding author address: Andreas Lorke, University of Constance, Limnological Institute, Mainaustrasse 252, D-78464 Constance, Germany.

E-mail: Andreas.Lorke@uni-konstanz.de
ADCP measurements evaluate the Doppler frequency shift of the backscattered signal to estimate the in-beam velocity of the scattering particles, the pulse-coherent mode evaluates the phase shift between the echoes of two subsequent pings, resulting in much higher measurement accuracy but shorter profiling ranges. With the assumption that the velocity is the same within the respective depth bins of each beam, a three-dimensional velocity vector can be constructed from the measured along-beam velocities of at least three beams.

A typical beam geometry is such that the beams are inclined by $20^{\circ}-30^{\circ}$ to the vertical and are symmetrically arranged within a horizontal plane projection (e.g., in a Janus configuration). Depending on the range from the instrument and the slant angle, the above assumption is equivalent to a velocity field that is horizontally homogenous on the scale of a few meters. For the mean velocity field this is usually an appropriate assumption for most flows in natural water bodies; however, it is not valid for turbulent velocity fluctuations, the data basis of the IDM. A detailed outline of the procedure to calculate a three-dimensional current vector based on the measured in-beam velocities of a three-beam ADCP is illustrated in Fig. 1. In analogy, for a four-beam ADCP the calculus is given, for in- 


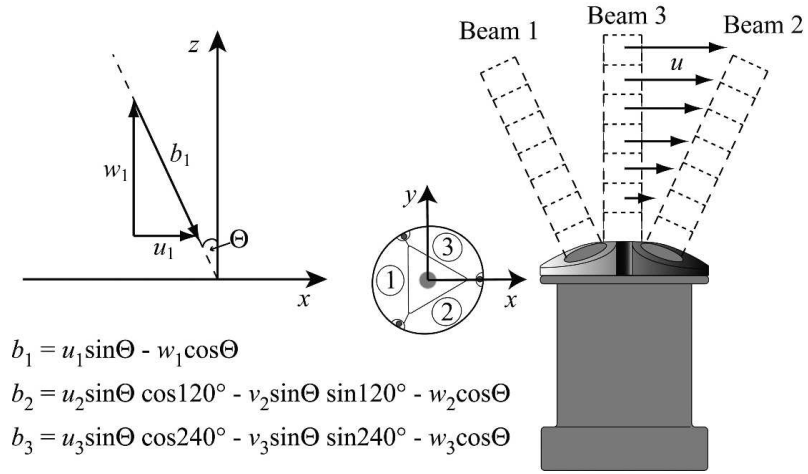

FIG. 1. Beam geometry and calculation of the horizontal and vertical velocity components $(u, v, w)$ from the measured in-beam velocities $\left(b_{1}, b_{2}, b_{3}\right)$ for a three-beam acoustic Doppler current profiler (such as the Nortek NDP). Whereas the derivation is exemplified for beam 1 , the respective derivations for beams 2 and 3 can be obtained by rotating the coordinate system by $120^{\circ}$ and $240^{\circ}$ in the horizontal $(x-y)$ plane. An unambiguous threedimensional velocity vector $(u, v, w)$ for each depth cell can be estimated only by assuming horizontal homogeneity of the flow field $\left(u_{1}=u_{2}=u_{3}, v_{1}=v_{2}=v_{3}, w_{1}=w_{2}=w_{3}\right)$.

stance, in Lu and Lueck (1999a). For the same instrument, it was shown by Lu and Lueck (1999b) that the statistical properties of the measured in-beam velocity fluctuations can be used to estimate selected Reynolds stresses as well as a term that is related to the turbulent kinetic energy (TKE) density. This procedure, known as the variance method, was applied to a variety of oceanic systems (e.g., Lohrmann et al. 1990; Stacey et al. 1999a,b; Rippeth et al. 2002). The application of the variance method, however, is restricted to ADCPs with at least four beams, as well as to highly energetic systems, such as tidal channels.

Thus, assuming an appropriate resolution and signalto-noise ratio, ADCPs in principle allow measuring the in-beam components of the turbulent velocity fluctuations. However, due to the geometrical spreading of the acoustic beams it cannot be assumed that each beam measures the same fluctuation. Hence the calculus, which provides the three-dimensional velocity vector based on the measured in-beam velocities (Fig. 1), fails and will result in incorrect estimates of the directions and magnitudes of the velocity fluctuations. Under the conditions of homogeneous turbulence within the beam area, it can be assumed that the statistical properties of the velocity fluctuations, such as their spectral energy distribution, are the same for all beams. The spectral properties also remain unaltered by the linear transformation from beam to orthogonal coordinates, and the IDM can be applied within the uncertainty of the unknown orientation of the velocity fluctuations relative to the mean flow.
The usefulness and limits of this approach are discussed in this paper by comparing dissipation rate estimates using the IDM from two different ADCPs with simultaneously measured dissipation rates from temperature microstructure profiles. The measurements were conducted in the low-energetic environment of a bottom boundary layer in a lake in the framework of an experiment focusing on the impact of bottom boundary turbulence on the diffusive flux of oxygen at the sediment-water interface (Lorke et al. 2003).

\section{Study site and instrumentation}

The measurements were carried out for $25 \mathrm{~h}$ on $13-$ 14 August 2002 in the bottom boundary layer of Lake Alpnach, a medium-sized subbasin of Lake Lucerne in central Switzerland. Lake Alpnach has an elliptical shape with axes lengths of 5 and $1.5 \mathrm{~km}$ and a maximum depth of $34 \mathrm{~m}$. The strongly pronounced seiching (Münnich et al. 1992; Gloor et al. 1994), resulting from daily winds along its main axis and the associated periodic nature of bottom boundary layer turbulence (Lorke et al. 2002), makes Lake Alpnach an ideal site for the present study.

Two ADCPs were deployed on the lake bottom, and temperature microstructure and conductivity-temperature-depth (CTD) profiles were measured on a regular time scale. All instruments were operated within a radius of about $100 \mathrm{~m}$ at a site close to the center of the lake at a local depth of $32.2 \mathrm{~m}$.

\section{a. ADCP measurements}

A four-beam, 614-kHz RDI Workhorse ADCP (RD Instruments) was deployed directly onto the sediment facing upward. The pulse-coherent mode (RDI mode 5) with a bin size of $0.1 \mathrm{~m}$ was used, enabling a measurement range from 0.6 to $8.1 \mathrm{~m}$ above the sediment (31.6-24.1-m depth). Groups of three pings were averaged internally, and the averaged data were recorded every $2 \mathrm{~s}$. In addition, a three-beam, 1.5-MHz Nortek NDP (Nortek AS, Norway) was mounted on a tripod facing downward, resolving the range from 0.05 to $2.2 \mathrm{~m}$ above the sediment (32.15-30-m depth) with a vertical bin size of $0.04 \mathrm{~m}$. The NDP was operated in highresolution (pulse coherent) mode and recorded the average of six individual pings every 9 s. Special data conversion software (A. Lohrmann, Nortek AS, 2002, personal communication) enabled an increase of the velocity precision by a factor of 10 for the Nortek NDP to $0.1 \mathrm{~mm} \mathrm{~s}^{-1}$, as compared to the standard value of 1.0 $\mathrm{mm} \mathrm{s}^{-1}$ for both instruments. 


\section{b. Temperature microstructure and CTD measurements}

Temperature microstructure profiles were measured with a pair of FP07 fast-response thermistors (Thermometrics) on an adapted SEABIRD SBE-9 CTD profiler (Sea-Bird Electronics, Inc.) (Kocsis et al. 1999). The probe was operated freely sinking with a speed of 8 $\mathrm{cm} \mathrm{s}^{-1}$ from 20-m depth to about $0.15 \mathrm{~m}$ above the sediment. The sampling frequency was $96 \mathrm{~Hz}$, and profiles were collected every $15 \mathrm{~min}$, resulting in 96 profiles in total and leaving enough time between subsequent casts for profiler-induced turbulence to be advected away from the sampling site (Jonas et al. 2003).

A SEABIRD SBE-19 CTD was used to measure profiles of temperature, conductivity, dissolved oxygen, $\mathrm{pH}$, and light transmissivity (at $660 \mathrm{~nm}$ ) throughout the entire water column every $2 \mathrm{~h}$.

\section{Data processing and analysis}

\section{a. Temperature microstructure method}

The temperature microstructure method (TMM) is based on the existence of a universal form of the highfrequency wavenumber spectrum of temperature or temperature gradient fluctuations in homogeneous and isotropic turbulence. The theoretical spectrum was estimated by Batchelor (1959), and its one-dimensional form has been validated by a variety of laboratory and field experiments (e.g., Grant et al. 1968). The viscousconvective and diffusive subranges of the one-dimensional temperature fluctuation spectrum $\Phi^{T}$ as a function of the scalar (vertical) wavenumber $k_{3}$ can be described by

$$
\Phi^{T}\left(k_{3}\right)=\sqrt{\frac{q}{2}} \frac{\chi}{k_{B} D} x\left(e^{-\left(1 / 2 x^{2}\right)}-x \int_{x}^{\infty} e^{-\left(1 / 2 x^{\prime 2}\right)} d x^{\prime}\right),
$$

where $x$ denotes the dimensionless wavenumber $x=$ $(2 q)^{1 / 2} k_{3} / k_{B}$ with the Batchelor wavenumber $k_{B}=$ $\left(\varepsilon / \nu D^{2}\right)^{1 / 4}$ and the Batchelor constant $q(\chi$ is the dissipation rate of temperature variance, and $D$ and $\nu$ are the molecular diffusivity of heat and the kinematic viscosity, respectively).

The typically low dissipation rates, which are often below the detection limit of oceanographic shear probes, in conjunction with short profiling ranges, allowing for a slow profiling speed, favor the temperature microstructure or Batchelor method over the profiling shear probes in lakes. The shortcomings of this method, arising from the limited response time of the thermistor (Gregg 1999), can usually be neglected under such con- ditions (Kocsis et al. 1999). Fitting measured temperature spectra to Eq. (1) has become a standard method for estimating $\varepsilon$ in lakes (e.g., Dillon et al. 1981; Luketina and Imberger 2001; Jonas et al. 2003). The temperature fluctuations are usually measured using slowly sinking or uprising profilers equipped with fastresponse thermistors. Taylor's (1938) frozen field hypothesis is used to transform the respective temperature fluctuation spectra from the frequency to the wavenumber domain using the profiling speed.

The measured temperature microstructure profiles were segmented using fixed and overlapping segmentation lengths of 512 data points, corresponding to $\sim 45$ $\mathrm{cm}$ long profile sections. Within these segments the profiler drop rate was sufficiently steady, and the respective fluctuation spectra in the wavenumber domain were fitted to Eq. (1) with $\varepsilon$ and $\chi$ as the fitting parameters. The probe, the data acquisition, and the data processing procedure have been described in detail in Kocsis et al. (1999) and Jonas et al. (2003).

\section{b. Inertial dissipation method}

Similar to the TMM, the inertial dissipaion method (IDM) is based on fitting measured fluctuation spectra to their respective theoretical and universal forms, however, not within the high-frequency dissipation range but within the inertial subrange of the wavenumber spectrum. There, the spectra often show the characteristic $k^{-5 / 3}$ slope as a function of the wavenumber $k$ (Tennekes and Lumley 1973). Although it was shown that fluctuation spectra of scalar properties like temperature, suspended solid, or chlorophyll concentrations follow this characteristic slope (Soulsby et al. 1984; McPhee 1994; Lee et al. 2003), the lack of universal scaling constants constrained the application of this method to measured spectra of the three-dimensional velocity fluctuations. In homogenous turbulent flow and with the assumption of isotropy, the diagonal components $\Phi_{i}^{K}(i=1, \ldots, 3)$ of the associated spectrum tensor can be separated:

$$
\Phi_{i}^{K}=\alpha_{i} \varepsilon^{(2 / 3)} k_{i}^{-(5 / 3)} .
$$

The index $i$ refers to the along-stream $(i=1)$ and crossstream $(i=2,3)$ components of the spectrum tensor, and $\alpha_{\mathrm{i}}$ refers to the one-dimensional Kolmogorov constant $\alpha_{K}\left(\alpha_{1}=\alpha_{K}, \alpha_{2}=\alpha_{3}=4 / 3 \alpha_{K}\right)$. By applying Taylor's hypothesis $\Phi_{i}^{K}$ is calculated from measured time series of the three-dimensional velocity, and thus $\varepsilon$ is estimated by spectral fitting (e.g., Gross and Nowell 1985; Dewey and Crawford 1988; McPhee 1998; Bertuccioli et al. 1999).

Since ADCPs do not resolve the directions of the 


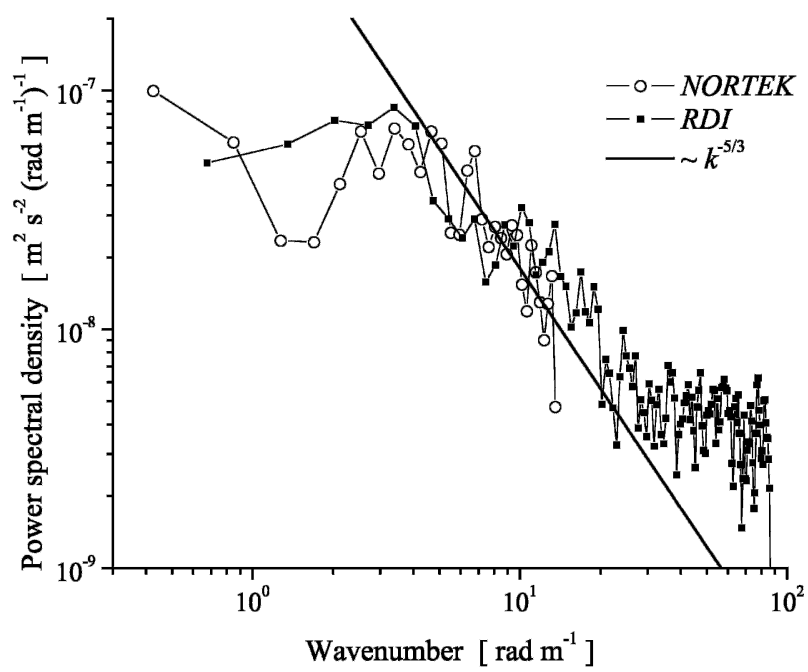

FIG. 2. Wavenumber spectra of the in-beam velocities measured simultaneously by the RDI ADCP and the Nortek NDP. The spectra were calculated over time periods of 19 (RDI) and 17 (Nortek) min, respectively, and averaged over all beams at a height of $1 \mathrm{~m}$ above the sediment. The straight line shows a $k^{-5 / 3}$ inertial subrange slope.

turbulent velocity fluctuations, along-stream and crossstream velocity spectra cannot be separated. Thus the isotropy constant $\alpha_{i}$ in Eq. (2) remains undetermined within the range $\alpha_{K} \leq \alpha_{i} \leq 4 / 3 \alpha_{K}$. Using spectral fitting [Eq. (2)] leads to an uncertainty for the dissipation rate estimation of up to a factor of $(4 / 3)^{-3 / 2} \approx 0.65$, which is well within the range of uncertainties of conventional methods (e.g., Peters and Gregg 1988; Yamazaki and Osborn 1990).

Segments of the measured time series of the velocities at all depths were first analyzed for the mean current direction and speed. Wavenumber spectra were calculated from the measured in-beam velocities and averaged over the three and four beams of the Nortek NDP and RDI ADCP, respectively. Segment sizes were 128 samples (19.2 min) for the Nortek and 512 samples (17 min) for the RDI. If the spectra exhibited an inertial subrange, a straight line representing a $-5 / 3$ slope was fitted to a double logarithmic spectrum plot, and $\varepsilon$ was estimated by rearranging Eq. (2), using a Kolmogorov constant $\alpha_{K}=1.56 \times 18 / 55$ for the streamwise spectral component (Wolk et al. 2002; Jonas et al. 2003). The particular choice of the isotropy constant results in the estimation of an upper limit for $\varepsilon$ due to the unknown orientation of the spectrum with respect to the mean flow. Sample spectra from both ADCPs are shown in Fig. 2. The wavenumber range used for the fitting procedure was adjusted manually for each spectrum according to the existence of an inertial subrange.

Alternatively, an automated procedure was em- ployed to perform the spectral fitting. This procedure, which was applied to fixed frequency ranges (from $9 \times$ $10^{-4}$ to $3 \times 10^{-2} \mathrm{~Hz}$ for the Nortek and from $1 \times 10^{-3}$ to $4 \times 10^{-2} \mathrm{~Hz}$ for the RDI), estimated $\varepsilon$ based on Eq. (2) by

$$
\varepsilon=\exp \left\langle\ln \left(\frac{\Phi^{K}}{\alpha_{K}(\omega / U)^{-5 / 3}}\right)^{3 / 2}\right\rangle
$$

with $\omega$ and $U$ being frequency and mean current speed and \langle\rangle denoting averaging over different frequencies in the logarithmic domain. A fixed frequency limit was chosen in favor of an upper wavenumber limit, since it was assumed that instrument noise is the most relevant process limiting the high-wavenumber resolution of the measurements and that this noise can be described more appropriately in the frequency domain.

Besides instrumental noise, the high-wavenumber response of the ADCP is limited by the system spatial resolution (Zedel and Hay 1999). The maximum wavenumber resolved by the instrument is $2 \pi /(2 d)$, with $d$ being the width of the acoustic beam in the particular depth bin. Given the transducer diameters of 0.043 and $0.078 \mathrm{~m}$ for the RDI and Nortek, respectively, and assuming a beam-spreading angle of $1.5^{\circ}$, the corresponding wavenumbers are $43-11 \mathrm{rad} \mathrm{m}^{-1}$ for the RDI and 78-30 $\mathrm{rad} \mathrm{m}^{-1}$ for the Nortek, depending on distance from the instrument. Whenever the wavenumber of the noise-limiting frequency exceeded the wavenumber of the system spatial resolution, the latter was used during the automated fitting procedure.

\section{Results}

The averaged current speeds and directions measured by both ADCPs are shown in Fig. 3. During the 25-h observation the speeds varied between 1 and 30 $\mathrm{mm} \mathrm{s}^{-1}$ and covered a bit more than one complete cycle of internal seiching with a period of about $18 \mathrm{~h}$. The regular seiching pattern was slightly disturbed by a moderate wind event at the end of the measurements. The main current directions (northeast and southwest, respectively) correspond to the alignment of the main axes of the lake. Although the current directions measured by both instruments agreed almost perfectly, systematic differences were found in the current speeds. As shown in Fig. 3, the differences in the measured current speeds depended on the current direction. Whereas during the northeast currents [1800-0600 local time (LT)] the speeds measured by both instruments agreed reasonably well, systematic differences of $\sim 4$ $\mathrm{mm} \mathrm{s}^{-1}$ (up to $60 \%$ ) were detected at the beginning and at the end of the 25-h observation (southwest current 


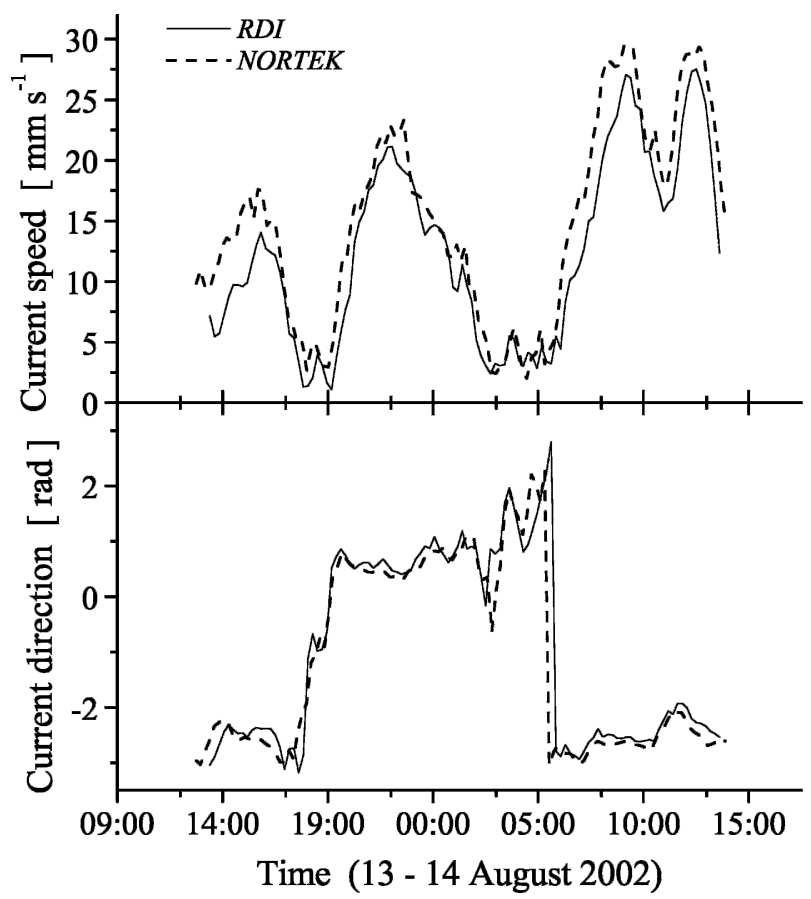

FIG. 3. Time series of the averaged current (top) speeds and (bottom) directions measured by the RDI ADCP and the Nortek NDP during the entire period of measurements at a height of $1 \mathrm{~m}$ above the sediment. The current direction is given in rad from north.

direction). The higher velocities were measured by the Nortek. Since the form of the current profiles also differed significantly during these periods (not shown), it cannot be excluded that the measured differences were real and could be attributed to topography-induced variations of the local flow field.

A comparison of the TMM-based dissipation rates with the IDM estimates from the two ADCPs is shown in Fig. 4. In general, the turbulence followed the same dynamics as the current velocity, resulting in a periodic structure. However, as shown by Lorke et al. (2003) and discussed in more detail in Lorke et al. (2002), the turbulent dissipation at some distance from the sediment lags behind the current velocity due to the seichinginduced periodicity of the flow. The estimated time lag at a height of $1 \mathrm{~m}$ above the sediment was between 1.5 and $2 \mathrm{~h}$ (Lorke et al. 2002).

The TMM-based dissipation estimates at a height of $1 \mathrm{~m}$ above the sediment varied over three orders of magnitude between $10^{-11}$ and $2 \times 10^{-8} \mathrm{~W} \mathrm{~kg}^{-1}$ throughout the seiching period. The IDM-based $\varepsilon$ values from the two ADCPs followed the same dynamics as the TMM-based dissipation estimates, and the correspondence between both methods was excellent (Fig. 4). An inertial subrange was observed in the velocity

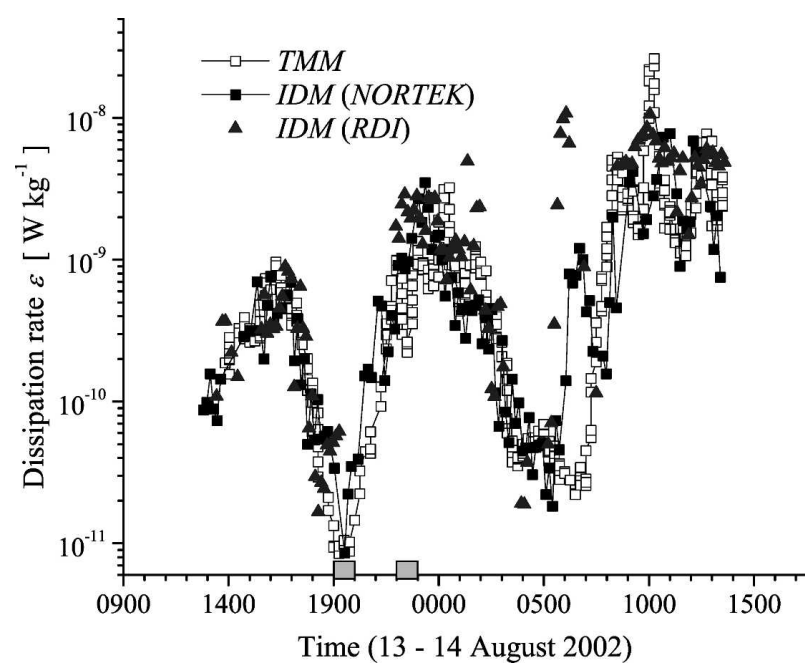

FIG. 4. Time series of the turbulent kinetic energy dissipation at a height of $1 \mathrm{~m}$ above the sediment estimated from TMM and from the RDI ADCP and the Nortek NDP by using the IDM. For the latter, only those estimates were shown for which the spectra showed a pronounced inertial subrange, as illustrated in Fig. 2 (68\% and $79 \%$ of the considered velocity spectra from the RDI and the Nortek, respectively). The two gray bars on the time axis indicate the averaging intervals for the dissipation profiles shown in Fig. 5.

spectra almost throughout the entire measurement period, although it was less pronounced at the rising flanks of the current speed (Fig. 4). A major discrepancy between the TMM- and IDM-based dissipation estimates was found on the morning of 14 August (0600-0800 LT), when high dissipation rates were measured by both ADCPs, whereas the TMM-based dissipation rate remained low.

The vertical structure of the dissipation rate is exemplified in Fig. 5, which shows dissipation profiles from all three instruments for periods of "strong" (Fig. 5a) and "weak" (Fig. 5b) turbulence, respectively. In contrast to the dissipation time series shown in Fig. 4, the IDM-based dissipation rates were estimated using an automated procedure by fitting a $k^{-5 / 3}$ slope to the spectra, regardless of whether or not they matched the Kolmogorov form.

The "strong" turbulence profile showed a pronounced bottom boundary layer within the lowest $2 \mathrm{~m}$ above the sediment, with turbulent dissipation rates increasing by more than two orders of magnitude. The bulk dissipation above, measured by the TMM, did not show much dynamics, with very weak dissipation rates between $10^{-11}$ and $10^{-10} \mathrm{~W} \mathrm{~kg}^{-1}$.

By comparing the two profiles, it becomes obvious that in this example the noise level or the detection limit of the automated estimation procedure for the RDIbased dissipation rate was about $4 \times 10^{-10} \mathrm{~W} \mathrm{~kg}^{-1}$, 


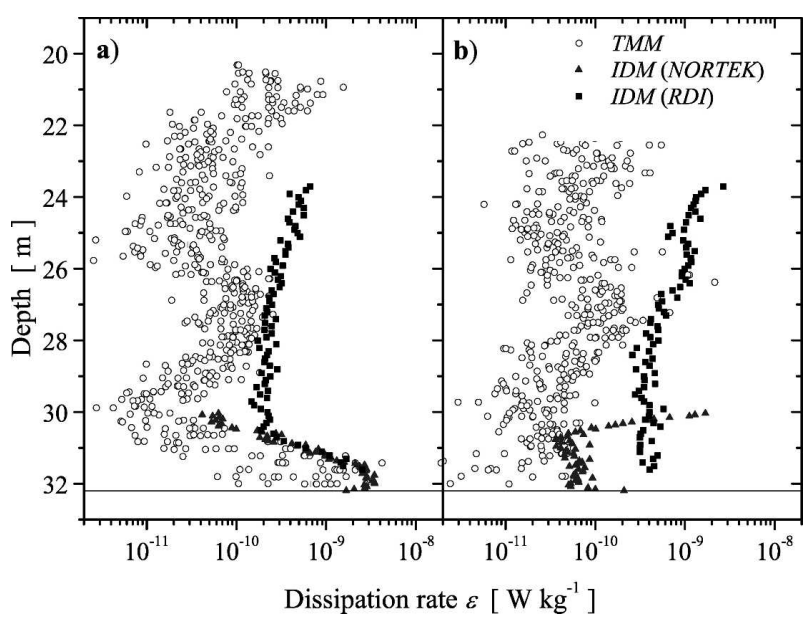

FIG. 5. Profiles of dissipation rates for (a) strong and (b) weak turbulence. The two profiles were averaged between 2200-2300 and 1900-2000 LT (13 Aug 2002), respectively. The respective times are indicated in Fig. 4. In contrast to Fig. 4, the inertial dissipation method was applied in an automated manner, regardless of whether a pronounced inertial subrange was present in the spectra.

slightly increasing with distance from the instrument. Hence all dissipation rate estimates from the RDI ADCP in Fig. 5b are noise only. The agreement of $\varepsilon$ from both ADCPs was excellent whenever the noise threshold was exceeded (29-30-m depth in Fig. 5a). Comparison with Fig. 4, however, reveals that this noise level was not constant throughout the measurement period. The noise level of the velocity measurements can be assumed to be fixed in the frequency domain, and the transformation to the wavenumber domain, using varying average current speeds, resulted in nonconstant noise thresholds of the dissipation estimates.

The dissipation rate estimates from the Nortek NDP showed some artifacts at distances close to the instrument (29-30-m depth in Fig. 5b). The different noise levels of the two ADCPs were related to the different acoustic frequencies and to the fact that the precision of the measured velocities from the Nortek NDP was one order of magnitude better compared to that of the RDI ADCP. The latter was probably more important, since the measured in-beam velocities were close to the standard instrument resolution of $1.0 \mathrm{~mm} \mathrm{~s}^{-1}$.

The system spatial resolution limited the highwavenumber response of the measurements only at depth bins farther away from the instrument. The corresponding maximum wavenumbers were 35 (RDI) and $47 \mathrm{rad} \mathrm{m}^{-1}$ (Nortek) for the spectra shown in Fig. 2. Because of the lower sampling frequency and the smaller transducer size, the Nortek spectra were not affected by the system spatial resolution. For the RDI, however, the automated fitting procedure adjusted the upper wavenumber limit depending on the distance from the instrument.

\section{Discussion}

The comparison of the dissipation rate estimates in the bottom boundary layer using the two different measurement techniques and three different instruments was limited by two general constraints. (i) For technological reasons the application of the IDM to ADCP data holds the lack of a properly defined isotropy constant $\alpha_{\mathrm{i}}$ in Eq. (2). (ii) Both methods, the TMM and the IDM, rely on the conditions of homogeneous and isotropic turbulence, which are not necessarily fulfilled for periodically forced bottom boundary layers. Further, considering the highly intermittent turbulence in natural waters (e.g., Baker and Gibson 1987), the correspondence of the dissipation rate estimates, using the two different techniques and three different instruments, was excellent. It is important to note that the two techniques differ fundamentally, not only in measurement principle (direct measurement of the velocity fluctuations versus associated temperature fluctuations) but also in wavenumber range under consideration. Whereas the TMM is most sensitive to the diffusive cutoff in the temperature fluctuation spectrum at the highest wavenumbers $\left(k>10^{2} \mathrm{rad} \mathrm{m}^{-1}\right)$, the IDM relies on the existence of an inertial subrange at wavenumbers that are at least one order of magnitude smaller.

As explained in the introduction, the application of the IDM to ADCP data has the uncertainty of the undefined isotropy constant $\alpha$ in Eq. (2) resulting from the unresolved directional information of the velocity fluctuations. The use of $\alpha$ for the streamwise fluctuation spectrum would result in the estimate of an upper limit for the dissipation rate $\varepsilon_{*}$ with the "true" dissipation rate $\varepsilon$ between

$$
\left(\frac{4}{3}\right)^{-(3 / 2)} \varepsilon_{*} \leq \varepsilon \leq \varepsilon_{*} .
$$

This range results from the difference between the isotropy constants $\alpha$ for the streamwise ( $\left.\alpha=\alpha_{K}\right)$ and crossstream-wise $\left(\alpha=4 / 3 \alpha_{K}\right)$ wavenumber spectra [Eq. (2)]. The actual factor within this given range, however, is not completely random but is biased by the orientation of the ADCP toward the mean flow. Figure 6 shows a direct comparison of $\varepsilon_{*}$ estimated from the Nortek NDP with the TMM-based dissipation rate (the figure reproduces the corresponding data from Fig. 4). Although the two independent estimates are linearly correlated over the entire range of the measurements, the 


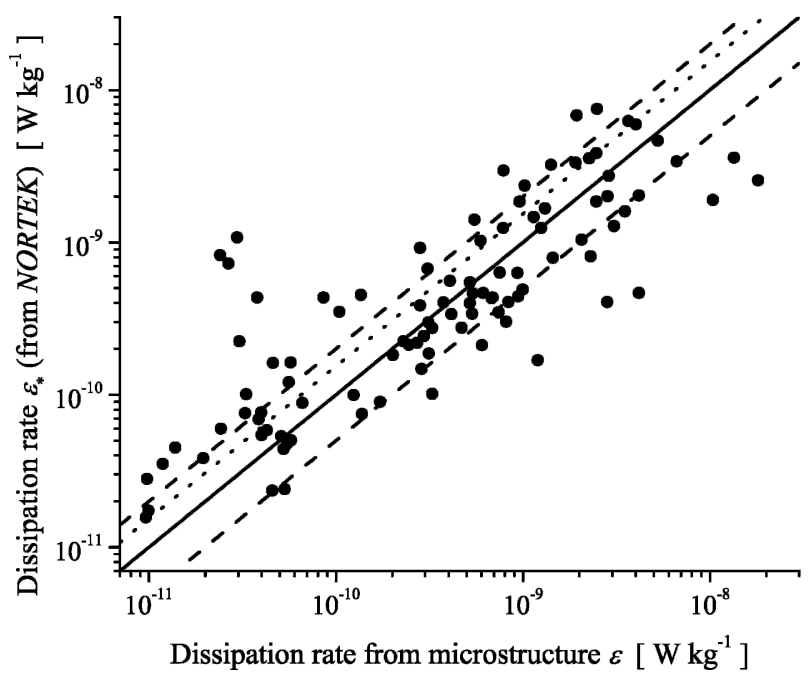

FIG. 6. Direct comparison of the dissipation rate $\varepsilon_{*}$ estimated using the IDM for the Nortek NDP with the temperature microstructure for the data shown in Fig. 4. The solid line shows a 1:1 relationship, whereas the dashed lines encompass an uncertainty factor of 2 , enclosing $60 \%$ of the dissipation estimates. The dotted line shows the relationship $\varepsilon_{*}=(4 / 3)^{3 / 2} \times \varepsilon$. According to Eq. (4), the expected range of the IDM-based estimates should be between the solid and the dotted lines.

large scatter does not allow the estimation of a bias, and $\varepsilon_{*} \approx \varepsilon$ seems to be the best first-order approximation. A linear regression of the logarithmic dissipation rates results in a slope of 0.99 . The range of uncertainty encompassed by Eq. (4) is shown in Fig. 6. This range is small compared to the overall uncertainty of the dissipation estimates resulting from the intrinsic intermittency in natural waters $\left(\sigma_{\ln \varepsilon} \approx 0.5 \ldots 2\right.$; Baker and Gibson 1987), which is usually expressed as an uncertainty factor of 2 (Kocsis et al. 1999).

\section{Conclusions}

The application of the inertial dissipation method (IDM) to ADCP velocity data is not a straightforward approach due to the missing directional information of the measured fluctuation spectra. However, a comparison of dissipation rates measured simultaneously by the conventional "profiling technique" (temperature microstructure) and by two different ADCPs revealed good agreement between both methods. The discrepancies are reasonable and similar to those found in other experiments that compare estimates of dissipation rates using two or more different techniques (e.g., Moum et al. 1995; Kocsis et al. 1999; Gregg 1999).

The measurements were conducted in the relatively low energetic bottom boundary layer of a lake. There was strong evidence that the dissipation rate estimates, at least from the RDI ADCP, were limited by instrument resolution. Hence it can be expected and should be proven in future experiments that the method performs at least as robustly in more energetic environments such as the coastal ocean or estuaries. A prerequisite, however, is the use of the pulse-to-pulse coherent operation mode of the ADCP, which enables higher-accuracy velocity measurements although restricting the measurement range to a few meters, depending on the acoustic frequency.

Because of its limited profiling range and accuracy, the described method cannot replace conventional turbulence measurements using vertical profiling techniques. However, as a supplementary method, the IDM offers the great advantage of potential long-term and autonomous operation. Furthermore, this method provides a remote sensing approach for simultaneously measuring dissipation and vertical current shear, the driving force of turbulence. Considering the future development of appropriate fitting procedures, as proposed for Batchelor curve fitting (e.g., Ruddick et al. 2000), the relatively simple algorithm of the inertial dissipation method promises great potential for fully automated data processing routines.

Acknowledgments. Many thanks are passed on to M. Schurter for his great support in the field. The constructive criticism of three reviewers significantly improved the clarity of the manuscript. D. McGinnis kindly corrected the English. The work was financially supported by the Swiss National Science Foundation (Grants 2000067091.01 and 200020-103827.1) and by EAWAG.

\section{REFERENCES}

Baker, M. A., and C. H. Gibson, 1987: Sampling turbulence in the stratified ocean: Statistical consequences of strong intermittency. J. Phys. Oceanogr., 17, 1817-1836.

Batchelor, G. K., 1959: Small-scale variation of convected quantities like temperature in turbulent fluid. J. Fluid Mech., 5, 113-133.

Bertuccioli, L., G. I. Roth, J. Katz, and T. R. Osborn, 1999: A submersible particle image velocimetry system for turbulence measurements in the bottom boundary layer. J. Atmos. Oceanic Technol., 16, 1635-1646.

Dewey, R. K., and G. B. Crawford, 1988: Bottom stress estimates from vertical dissipation rate profiles on the continental shelf. J. Phys. Oceanogr., 18, 1167-1177.

Dillon, T. M., J. G. Richman, C. G. Hansen, and M. D. Pearson, 1981: Near-surface turbulence measurements in a lake. $\mathrm{Na}$ ture, 290, 390-392.

Gloor, M., A. Wüest, and M. Münnich, 1994: Benthic boundary mixing and resuspension induced by internal seiches. Hydrobiologia, 284, 59-68.

Grant, H. L., B. A. Hughes, W. M. Vogel, and A. Moillet, 1968: The spectrum of temperature fluctuations in turbulent flow. J. Fluid Mech., 34, 423-442. 
Gregg, M. C., 1999: Uncertainties and limitations in measuring $\varepsilon$ and $\chi_{\mathrm{T}}$. J. Atmos. Oceanic Technol., 16, 1483-1490.

Gross, T. F., and A. R. M. Nowell, 1985: Spectral scaling in a tidal boundary layer. J. Phys. Oceanogr., 15, 496-508.

Jonas, T., A. Stips, W. Eugster, and A. Wüest, 2003: Observations of a quasi shear-free lacustrine convective boundary layer: Stratification and its implications on turbulence. J. Geophys. Res., 108, 3328, doi:10.1029/2002JC001440.

Kocsis, O., H. Prandke, A. Stips, A. Simon, and A. Wüest, 1999: Comparison of dissipation of turbulent kinetic energy determined from shear and temperature microstructure. J. Mar. Syst., 21, 67-84.

Lee, G.-H., W. B. Dade, C. T. Friedrichs, and C. E. Vincent, 2003: Spectral characteristics of bed shear using suspendedsediment concentrations in a wave-current boundary layer. $J$. Geophys. Res., 108, 3208, doi:10.1029/2001JC001279.

Lohrmann, A., B. Hackett, and L. P. Roed, 1990: High resolution measurements of turbulence, velocity and stress using a pulse-to-pulse coherent sonar. J. Atmos. Oceanic Technol., 7, 19-37.

Lorke, A., L. Umlauf, T. Jonas, and A. Wüest, 2002: Dynamics of turbulence in low-speed oscillating bottom-boundary layers of stratified basins. Environ. Fluid Mech., 2, 291-313.

—, B. Müller, M. Maerki, and A. Wüest, 2003: Breathing sediments: The control of diffusive transport across the sediment-water interface by periodic boundary-layer turbulence. Limnol. Oceanogr., 48, 2077-2085.

Lu, Y., and R. G. Lueck, 1999a: Using a broadband ADCP in a tidal channel. Part I: Mean flow and shear. J. Atmos. Oceanic Technol., 16, 1556-1567.

— and - 1999b: Using a broadband ADCP in a tidal channel. Part II: Turbulence. J. Atmos. Oceanic Technol., 16, $1568-1579$.

Luketina, D. A., and J. Imberger, 2001: Determining turbulent kinetic energy dissipation from Batchelor curve fitting. J. Atmos. Oceanic Technol., 18, 100-113.

McPhee, M. G., 1994: On the turbulent mixing length in the oceanic boundary layer. J. Phys. Oceanogr., 24, 2014-2031.

- 1998: An inertial dissipation method for estimating turbu- lent flux in buoyancy-driven, convective boundary layers. $J$. Geophys. Res., 103, 3249-3255.

Moum, J. N., M. C. Gregg, R. C. Lien, and M. E. Carr, 1995: Comparison of turbulence kinetic energy dissipation rate estimates from two ocean microstructure profilers. J. Atmos. Oceanic Technol., 12, 346-366.

Münnich, M., A. Wüest, and D. Imboden, 1992: Observation of the second vertical mode of the internal seiche in an alpine lake. Limnol. Oceanogr., 37, 1705-1719.

Peters, H., and M. C. Gregg, 1988: On the parameterization of equatorial turbulence. J. Geophys. Res., 93, 1199-1218.

Rippeth, T. P., E. Williams, and J. H. Simpson, 2002: Reynolds stress and turbulent energy production in a tidal channel. $J$. Phys. Oceanogr., 32, 1242-1251.

Ruddick, B., A. Anis, and K. Thompson, 2000: Maximum likelihood spectral fitting: The Batchelor spectrum. J. Atmos. Oceanic Technol., 17, 1541-1555.

Soulsby, R. L., A. P. Salkield, and G. P. Le Good, 1984: Measurements of the turbulence characteristics of sand suspended by a tidal current. Cont. Shelf Res., 3, 439-454.

Stacey, M. T., S. Monismith, and J. R. Burau, 1999a: Measurements of Reynolds stress profiles in unstratified tidal flow. $J$. Geophys. Res., 104, 10 933-10949.

,-- , and $-1999 \mathrm{~b}$ : Observation of turbulence in a partially stratified estuary. J. Phys. Oceanogr., 29, 1950-1970.

Taylor, G. I., 1938: The spectrum of turbulence. Proc. Roy. Soc. London, 164A, 476-490.

Tennekes, H., and J. L. Lumley, 1973: A First Course in Turbulence. MIT Press, 300 pp.

Wolk, F., H. Yamazaki, L. Seuront, and R. G. Lueck, 2002: A new free-fall profiler for measuring biophysical microstructure. $J$. Atmos. Oceanic Technol., 19, 780-793.

Yamazaki, H., and T. Osborn, 1990: Dissipation estimates for stratified turbulence. J. Geophys. Res., 95, 9739-9744.

Zedel, L., and A. E. Hay, 1999: A coherent Doppler profiler for high-resolution particle velocimetry in the ocean: Laboratory measurements of turbulence and particle flux. J. Atmos. Oceanic Technol., 16, 1102-1117. 